\title{
Transgresser pour mettre en œuvre le droit d'accéder à une vie ordinaire
}

Anne-Charlotte TAILLANDIER, Université Artois, LEM UMR 8179 CNRS, France

Le destin des personnes atteintes de déficience mentale a longtemps été de vivre en établissements spécialisés et, selon les statistiques d'espérance de vie, de mourir avant les autres. Les progrès de la science et de l'hygiène ont mis fin à cette période et le vieillissement de ces populations, comme les regards nouveaux sur le handicap, a induit des questionnements inédits sur la condition de vie de ces derniers et les capacités d'accueil et de solidarité de la société.

Sachant qu' « en moins d'un demi-siècle, nos débats, nos représentations, nos conceptions de l'altérité ont si profondément évolué qu'il faut s'interroger» (Wieviorka 3), l'équipe de chercheurs $^{1}$ accompagne l'installation de jeunes, porteurs de la trisomie 21, en habitat ordinaire. Après la prise en charge précoce au sein de la famille et une scolarisation en milieu ordinaire, les parents, réunis en association, mettent en œuvre des projets et actions, visant à l'inclusion dans la société. Ils encouragent les jeunes à habiter en environnement ouvert et à rechercher un travail hors établissement et service d'aide par le travail (ESAT). L'opportunité d'une rénovation immobilière, réalisée par un bailleur social, en vue d'un habitat collectif intergénérationnel intelligent est saisie comme solution alternative vers une forme d'autonomie. Même si ce franchissement d'une autre barrière s'inscrit dans la continuité des conduites des parents, le départ du cocon familial, difficile pour tout jeune, revêt une acuité particulière avec le handicap. Le choix et l'organisation réfléchie d'un suivi, voire d'une guidance, structurés, associant familles et professionnels - de l'éducation, du sanitaire et du médico-social - et s'appuyant sur les NTIC, contribuent au développement du vivre ensemble et visent à donner une dimension réelle à cette démarche d'autonomie sécurisée.

L'objectif est d'analyser les possibilités, méthodes et moyens d'insertion de ces personnes diversement «en difficultés» dans le complexe architectural, social et technologique rénové au sein d'un quartier de cœur de ville moyenne, à revitaliser. Les logements sociaux, équipés de tablettes numériques, en liaison notamment avec le portail du complexe, bénéficient de divers services à domicile. Les jeunes y sont installés, depuis près de dix-huit mois, au début de l'étude, parmi des locataires seuls ou en couple avec enfants et des personnes retraitées non dépendantes. 
Les résultats présentés reposent sur l'exploitation d'une première série d'entretiens, menés auprès des parents, ainsi que sur les douze séances d'observation effectuées lors des différentes visites dans ce complexe d'habitat. Cette première phase d'enquête qualitative a également concerné le personnel accompagnant au sens large. Dans un deuxième temps, d'autres entretiens permettront de faire le point des apports, après plus de deux ans, dans l'immeuble. Des questions ouvertes, posées aux personnes trisomiques elles-mêmes, les inciteront à exprimer leur ressenti et leurs projets. Compte tenu de la richesse des récits recueillis, l'exploitation, toujours en cours, sera remise en perspective avec l'analyse de ces nouveaux matériaux.

L'usage, dans cet article, du terme «transgression» concerne tant le champ individuel que social et évoque le dépassement de certaines normes - plus administratives et organisationnelles que juridiques -, le franchissement plus ou moins conscient de limites, instaurées par des règles, appliquées sans nuances, des conventions, des représentations sociales, voire une incitation à les revisiter. Il s'agit d'analyser un processus, constitué d'un cumul de démarches, toutes orientées vers le même objectif et sous-tendues par une seule conviction : indépendamment de leurs performances, ces jeunes trisomiques « égaux en droits et en dignité » (Gardou et Poizat 21), doivent, en tant que personnes, trouver leur « place » et parvenir, par un accompagnement adapté, à exprimer comme tout un chacun leurs désirs, pour « prendre un rôle » (Kaufmann 200) dans la société.

Partant du vécu de l'annonce de la trisomie, le plus souvent après la naissance puisqu'il s'agit d'adultes, tous âgés de plus de vingt ans - l'étude porte sur les accompagnements parentaux divers et successifs de ces jeunes, depuis leur scolarité jusqu'à l'installation individuelle en appartement, en tant que locataires et la recherche d'un emploi. Les entretiens, priorisant le récit, permettent de retracer l'histoire familiale et la construction du parcours personnel des jeunes, en intégrant leurs choix et - pour la plupart qui travaille les modalités de l'insertion professionnelle ainsi que les difficultés, étapes et méthodes de la recherche de formations et/ou d'emploi pour tous.

Comment les représentations sociales, les politiques mal adaptées ou mal comprises et les manques de flexibilité, les insuffisances et les dysfonctionnements des structures et des institutions, conduisent-ils des parents à s'engager dans des choix et des pratiques souvent inattendus, non conformes aux normes collectives, parfois jugés choquants, voire provocateurs ? Quel hiatus existe-t-il entre les objectifs, les visées de ces parents, qu'ils considèrent être de l'intérêt de leurs enfants et les moyens et aides auxquels ils peuvent prétendre pour atteindre ces buts? 


\section{Une définition, des représentations du handicap}

Malgré la définition légale - et paradoxalement, en partie à cause - de la loi du 11 février 2005, le handicap reste une « construction sociale » au sens de Henri-Jacques Stiker (Corps). Ses représentations, comme les réactions qu'il suscite, sont multiples, touchant à la culture, l'identité mais aussi liées au droit, à la déclinaison institutionnelle des solidarités, à l'individualisme de notre époque et à l'économie. La loi 2005-102 «pour l'égalité des droits et des chances, la participation et la citoyenneté des personnes handicapées » évoque « toute limitation d'activité ou restriction de participation à la vie en société subie dans son environnement par une personne en raison d'une altération substantielle, durable ou définitive d'une ou plusieurs fonctions physiques, sensorielles, mentales, cognitives ou psychiques, d'un polyhandicap ou d'un trouble de santé invalidant ». Cette définition large englobe, en principe, toutes les situations de handicap mais renvoie au problème récurrent, qu'indiquait $\mathrm{P}$. Woods, cité par C. Duflot, d'« une limitation ou une modification des rôles sociaux que l'intéressé aurait souhaité tenir ou qu'on aurait attendu de sa part ».

Du fait de leur diversité et de la complexité, les situations de handicap sont mal connues, voire méconnues. Comme les personnes dans ces situations sont aussi différentes, ne sont pas ou ne se comportent pas «comme les autres », elles peuvent apparaître comme des intrus, des étrangers, au sens de G. Simmel, à la fois « dedans » et « dehors ».

De façon générale et quel qu'il soit, le handicap interpelle, choque, scandalise, parfois. Il fait peur parce qu'il rappelle des vulnérabilités contestées, voire niées. Il va à l'encontre de ce que valorise la société tant sur le plan esthétique qu'au regard des capacités intellectuelles et physiques et crée du découragement, car il constitue un obstacle qui souligne les impuissances. Les regards tantôt misérabilistes, tantôt porteurs de réactions narcissiques, peinent à considérer la personne pour elle-même. Le «moi » cherche à se protéger en activant des «mécanismes de défense» (Ionescu 45) ou recourt au «coping évitant» (Lecomte 53).

Comme ils coûtent à la collectivité et font partie de la «dette publique » au titre des politiques sociales, des institutions et des équipes concernées, les handicaps divers mobilisent la recherche médicale pour « limiter» en amont les naissances d'enfants handicapés. Etudes génétiques, dépistages in utero, mesures préventives diverses, y compris interruptions médicales de grossesse, etc. induisent des combinaisons d'examens, de technologies et d'interventions onéreuses qui font regretter aux parents certains choix budgétaires, pour qui des financements d'adaptation et de progrès seraient parfois plus conformes à l'intérêt général que la recherche systématique d'élimination. 
Dans le contexte de progrès et de spécialisations, les accompagnements des personnes handicapées se sont diversifiés tant au regard des activités - paramédicales, médico-sociales - que des fonctionnalités des technologies. Outre les problèmes d'accessibilité et de discrimination, le vieillissement de ces personnes a donné lieu à divers études et rapports. En septembre 2013, notamment, le Comité interministériel du Handicap a rappelé : «Chacun peut légitimement aspirer à être un travailleur, un citoyen, un conjoint, un ami, un passionné d'art ou de sport... et plus encore à être tout cela à la fois. Idéalement, il incombe à chaque individu d'en décider selon un principe d'autonomie ; c'est le rôle des pouvoirs publics de le permettre ». La littérature et plusieurs films ont incité, ces dernières années, à renouveler les regards et les approches. Le développement des environnements humains au travers des maisons départementales (MDPH), des commissions des droits et de l'autonomie (CDAPH) et des projets personnalisés, contribue à des prises de conscience mais beaucoup reste à faire : plus de $60 \%$ des entreprises, soumises à l'obligation d'employer des personnes handicapées, la respecte (RSE) mais le taux de chômage de ces populations est encore le double de celui des valides, pourcentage encore aggravé depuis la crise économique.

Dans le cas particulier des personnes trisomiques, il ressort des entretiens que les interprétations de ce que nous qualifions «transgressions» induisent, selon les acteurs parents, personnel médical et médico-social, enseignants, etc. - des significations différentes. Les représentations (Jodelet) sont influencées tant par les aspects matériels que par les projections, désirs, motivations : chacun ignorant, n'imaginant pas ou niant les contraintes supportées par l'autre et se sentant légitime, du fait des normes (administratives, professionnelles...) ou fondé à réclamer « une vie ordinaire ».

L'idée de transgression émerge le plus souvent à partir des divergences d'«interprétations ». La conviction que les catégorisations conduisent rapidement aux stéréotypes, voire aux préjugés et stigmates (Goffman), incitent certains parents, à « sortir du problème » en tentant de renouveler les concepts. Pour lutter contre les clichés, les peurs et l'ignorance, ils revisitent les discours et les pratiques et espèrent « déstabiliser l'ordre géométrique de notre univers normatif et de ses canons rhétoriques » (Gardou et Poizat 9). Refusant la conformité, ils réinterprètent les situations, cherchent à adapter les rôles, les organisations, les outils et les lieux : «L'interprétation intervient pour assigner un sens ou du sens à un certain type de texte ou d'acte. [...] On interprète des signes, des symptômes, des manifestations qui se caractérisent par un écart entre ce qui se montre et ce qui est là véritablement visé. [...] ce qui implique de disposer des normes valides de transposition d'un 
langage dans un autre, elle se présente comme une espèce de traduction » (Weiss). Il ne s'agit, en aucun cas, de détruire mais bien de « déconstruire » ou, comme l'explique Philippe Meirieu à propos de l'apprentissage, de «transgresser de façon constructive », donc de se départir d'une vision négative de la transgression. Dès le départ ou à un moment de la vie de leurs enfants, ces parents ont refusé la « logique de placement institutionnel, de prêt-à-porter, quand il conviendrait de penser en termes de trajectoires en mouvement, de sur-mesure » (Gardou et Poizat 12). Ils ont rappelé « le droit à une orientation adéquate » (Gossot et Mollo 114), c'est-à-dire déjà la mise en œuvre effective de la loi de 1975. Dès lors, ils veillent en permanence à augmenter le potentiel d'action des jeunes, pour détruire l'identité sociale traditionnelle $\mathrm{du}$ 《trisomique » et reconstruire une autre image de la déficience ou de la différence. La recherche des parents pour un accès à l'autonomie des jeunes handicapés se traduit par un souci constant d'élargir les choix que la société menace.

\section{Crise et complexité du " mettre au monde "}

Faire naître, «mettre au monde », est une «aventure » que vivent depuis des millénaires les être humains, « laissant le hasard du génotype déterminer les différences et les ressemblances, le singulier et le semblable, l'unique et le commun » (Fils-Trèves 1) ; c'est un événement, le plus souvent voulu et/ou désiré depuis le progrès des méthodes de contraception, la légalisation et la prise en charge de l'avortement, un choix réfléchi et délibéré, dans le cas des techniques biomédicales. Les innovations technologiques et l'évolution des repères qu'elles provoquent, transforment les regards, bousculent les représentations, remettent en cause les mentalités, font vaciller les valeurs et suscitent des débats.

L'arrivée d'un enfant handicapé induit, pour les parents et les proches, la perte de l'insouciance, des beaux rêves et des projets pour le bébé. Comme le rappelle Eléonore Laloux, jeune adulte trisomique, confrontés par nécessité à l' « aberration chromosomique », il leur faut « vivre avec » (9). Il se produit un brouillage : l'angoisse, la tristesse, la colère aveuglent et empêchent de faire «le deuil de l'enfant ordinaire » attendu. Comme « dans la plus simple des histoires, on projette toujours de soi, de son image, de sa stabilité, de son couple, de ses aspirations, on attend de lui beaucoup, surtout lorsqu'il est le premier» (Frydman 134), lorsque l'annonce injecte « le poison » (Laloux 7), ce sont les personnes dans leur totalité qui sont remises en cause : l'imaginaire s'effondre, le renoncement tarde à se construire, les « parents sont perdus » (13). 
Le choc est décrit diversement selon les personnes ; il apparaît sous forme de traumatisme, d'abattement, d'incrédulité, de sidération, etc. Mais, pour tous, les repères se dérobent et après la déchirure, dans un premier temps, le désespoir succède à l'anéantissement. C'est le «miroir brisé » (Sausse). L'annonce de la terrible nouvelle laisse chez chacun une marque indélébile : propos brefs, brusques, silences, évitements, interrogations sans réponses. Ce souvenir de l'annonce du handicap reste un des souvenirs les plus douloureux, notamment $\mathrm{du}$ fait des erreurs, du ton employé, du moment: "Pour beaucoup, les expressions du visage d'autrui et l'impression qu'elles ont laissée en eux sont ancrées dans la mémoire pour des années ou toute la vie » (Fromm 50). Beaucoup de temps a été nécessaire pour "émerger», dépasser les maladresses, les paroles malheureuses et répétitives.

Dans la plupart des entretiens, il reste de l'amertume quant au manque de dialogue, à l'isolement face à la perte de ressources et à l'incompréhension. Désemparés, démunis, les parents «médusés » (Sausse) ont le souvenir d'avoir été submergés par la peine, tantôt paralysés dans leur solitude, tantôt ballotés, en même temps que leur enfant, dans les auscultations, analyses, examens, interventions chirurgicales : «Tout a été très vite, puis on s'est retrouvés seuls dans la chambre, à attendre, sans savoir », dit une mère lors d'un entretien.

Ces moments de solitude - seul ou à deux - sont évoqués avec beaucoup d'émotion, le malaise du «pourquoi nous ? », mis à distance par les années et le vécu ultérieur, est resté sans réponse. A l'évocation de la mémoire familiale, le souvenir des perturbations de l'ensemble de la structure (fratrie, grands-parents, etc.) ressurgit. Si l'incompréhension et les explications parcellaires quant aux causes sont encore très présentes, il n'y a plus de culpabilisation, pas de recherche d'arguments ou d'excuses, les parents ont mesuré combien tout cela épuise et n'aide pas à progresser.

Les termes employés pour évoquer la commune angoisse pour l'avenir, l'effroi, la peur de l'inconnu varient de l'un à l'autre et les mentions des expériences de tentations diverses - déni, méfiance, surprotection, etc. - émaillent ponctuellement les récits. Certains évoquent leurs doutes, avant même la révélation du problème, d'autres se rappellent la solitude et des allées et venues précipitées et gênées. Après la découverte d'une anomalie in utero, des parents effondrés ressentent « le poids » des paroles/conseils (médecins, famille ou autres) pour ne pas laisser naître une personne «qui serait malheureuse », " rendrait son entourage malheureux $»$ : logique du système de dépistage plus orienté, selon eux, vers l'élimination que vers le soin et l'intervention, du fait que l'interruption de grossesse pour 
raison d' «affection grave incurable » est autorisée depuis la loi de 1975, jusqu'au dernier jour «à tout moment». En cas d'《échec de dépistage » et de découverte post-natale, aucun parent n'a évoqué l'idée d'abandon.

Dès le départ de la socialisation en crèche, à l'école, pour les loisirs, les parents luttent pour que les accompagnants ne se limitent pas à l'attendrissement immédiat, qui se transforme ensuite en refus, en fermeture lorsque le besoin d'engagement, les obligations, les contraintes se font sentir. Il s'agit pour eux de trouver les mots pour que les éducateursanimateurs parviennent à voir autre chose que «l'aspect 'archaïque' et déficitaire » (Prayez) des conduites de l'enfant. A ce moment, le handicap «n'habite pas encore » (Chiflet 21) la personne elle-même, trop jeune, insouciante ; il « est vécu naturellement» (Nuss 103). Ce sont les parents qui souffrent, se heurtent chaque jour aux barrières autant qu'aux limites, s'inquiètent de ne rien pouvoir anticiper. Ils savent à quel point le « regard du corps social, a pour conséquence de surhandicaper progressivement et insidieusement l'enfant, le réduisant à son handicap et l'y enfermant durablement » (103).

Très vite, le regard des autres, non plus des professionnels mais des «gens ordinaires » pèse lourdement : sensibles aux sourires et moqueries des autres enfants, selon le contexte et/ou leur lassitude, les parents communiquent, expliquent ou affectent de ne pas voir. Les allées et venues se multiplient, les séances et consultations régulières chez les spécialistes s'ajoutant aux trajets scolaires habituels. Alors que pour un enfant «pas comme les autres », les cris, le brouhaha des cours de récréation, les rythmes et vitesses de réaction sont inquiétants, mal compris ou font peur, la bienveillance est loin d'être toujours au rendezvous. Le poids des évaluations sur des parcours scolaires lents et difficiles, induirait vite un niveau de qualification inférieur à la moyenne, alors que certains parents soulignent que leur enfant savait lire avant le CP. Au fil de la scolarité, les frustrations des options fermées se multiplient et s'ajoutent au désagrément des salles d'attente et cabinets de thérapeutes. En marge de l'activité professionnelle parentale, la patience et les énergies sont mises à rude épreuve. Il ressort des souvenirs et des restitutions de paroles mémorisées par les proches, que les professionnels insistaient d'abord sur les manques, les retards et qu'il fallait souvent leur rappeler les progrès.

L'insertion est, dans ces conditions, d'autant plus difficile et les atteintes à la liberté de la personne handicapée, comme de son entourage, sont quotidiennes. «C'est tout qui est plus lent, plus compliqué... », résume un père qui évoque le cumul d'obstacles à franchir. La mobilisation permanente des réseaux, seul remède aux tentations de découragement et d'isolement, même quand elle s'avère opérante, est lente à se mettre en place et à réagir. Dans 
tout contexte - école, travail, habitat - pour continuer à vivre, dépasser la crise, les parents imposent la personne handicapée, insistent voire exigent qu'on lui trouve et/ou qu'on lui « fasse » une place : «partant du postulat que tout être humain, aussi démuni soit-il, a quelque chose à dire de sa position subjective, il faut s'intéresser à ce que l'enfant lui-même a à dire de son handicap » (Korff-Sausse, Vie, 89). Soucieux d'agir dans l'intérêt de l'enfant, ils protègent mais aussi le poussent, expliquent, argumentent, négocient. Au cours des démarches de tous types, ils se voient contraints de lutter, d'imposer, parfois de menacer. A tout moment, ils éprouvent le sentiment de «transgresser », en contestant, forçant, passant outre, pour « déverrouiller un placard » (Styron 57) dans lequel la société serait prompte à ranger les personnes handicapées.

\section{Contraintes relationnelles et « choix » de vie}

Dès la petite enfance, l'entourage cherche une représentation, une conceptionconstruction qui permette à l'enfant de prendre sa place. Cette vision différente passe par les paroles, les regards, d'autres touchers. C'est à tout instant que les parents travaillent à l'individuation, corrigent l'image du corps et gomment les stigmates de «l'identité trisomique », en expliquant à l'enfant son histoire et sa maladie, afin qu'il s'accepte tel qu'il est et qu'il progresse. Le besoin de poursuite de soi, mis à mal par la « différence », n'aide pas à trouver les mots. Il est plus facile de parler de ce que l'on connaît. L'envie de ressemblances se fait sentir, alors même que la trisomie a contribué à « détisser » les liens et la recherche d'un mimétisme physique ou psychique, par-delà la déficience chez un enfant difficile (au moins matériellement) à élever, ne facilite pas l'exercice de «l'impossible métier ». Malgré beaucoup d'efforts et d'attention et quelle que soit sa gentillesse, cet enfant "préoccupant» sème des doutes très fréquents et rappelle à ses parents qu'ils sont encore moins compétents. Pour sélectives et mesurées que soient les transmissions, elles se veulent suffisamment compréhensibles et explicites pour ne pas engendrer de secrets, eux-mêmes créateurs de culpabilité et d'angoisses. Les précisions et souci de nuances entendus en entretien renvoient à Simone Korff-Sausse: «L'idée de la dangerosité de la transmission s'impose de manière souvent insidieuse à ceux qui sont atteints d'un handicap ainsi qu'à leurs géniteurs » (Figures, 60).

Le travail des «mots » nécessite un « travail sur soi » (Cresson), sur les émotions, comme au sein de la famille. Il prend du temps, demande beaucoup de recul et de réflexion et peut poser problème au sein d'une fratrie. La mobilisation des énergies reste relative afin que tout ne tourne pas, pour chacun, autour de la personne handicapée. Le temps, les contraintes 
matérielles lourdes sont compensées par une plus forte intensité de relation, par une présence, des partages spécifiques avec chacun des autres frère(s) et/ou sœur(s). La singularité et les exigences des situations de handicap, plaçant les préoccupations de compensation et de prévention au premier plan, risquent de susciter des réactions et/ou des impressions de favoritisme. En présence de frères et/ou sœurs, les parents évoquent le problème comme un risque et se veulent très vigilants aux signes de frustrations, voire de jalousie ou d'isolement qu'ils pourraient discerner. Ils fondent leur système commun - mais nécessairement non égalitaire - d'éducation, sur la volonté de répondre aux besoins de chacun. Ils veillent à rendre le vécu des autres, le moins douloureux possible et respectent « la prise de rôle » (Kaufmann 200) de chacun car «le soutien et les soins envers les proches supposent l'élaboration et la justification des attachements et des détachements, au cours de processus intrapersonnels et interpersonnels » (Pennec 237). Certains expliquent, en partie par cette raison, leur décision de ne pas avoir d'autres enfants.

Au regard de l'intégration scolaire, des récits renvoient aux écrits de R. Scelles pour qui ce temps «peut être un temps maturatif pour chacun, pour peu que les adultes soient à l'écoute de ce que tous les enfants vivent» (123). Il s'agit d'une « mise à l'épreuve » des frères et sœurs appelés « à jouer souplement entre des mouvements d'identification et de différenciation entre pairs et leurs ressources pour affronter les réactions des enfants et des adultes vis-à-vis du handicap » (123).

Parfois, pour éviter le risque de surprotection, peut-être en « contre-investissement », les autres enfants semblent bénéficier d'une liberté plus grande : certains n'ont pas hésité à s'éloigner pour étudier, voire travailler à l'étranger. Cette attention particulière se double de la difficulté, pour les parents, de faire accepter par l'enfant trisomique que ses frère(s) et/ou sœur(s) se livre(nt) à des activités, qu'il ne peut pas faire. L'interrelation, toujours complexe, renvoie en permanence aux contraintes matérielles.

Ces tensions provoquent des mises en jeu «des valeurs et des normes » de chacun, génératrices de conflits au regard des «pratiques domestiques et familiales » et supposent « des ajustements négociés » (Pennec 237), qui s'avèrent d'autant plus difficiles en présence d' « injonctions professionnelles ». Comme dans le soin aux personnes âgées, « les réactions et suggestions des soignants » dans l'accompagnement du handicap «sont vécues comme d'amères déconvenues » : explications minimales, voire absence d'information, évitements. Cela peut aller au rejet, lorsque les parents posent trop de questions, veulent comprendre et/ou intervenir dans les décisions, refusent d'être dépossédés de leur enfant. L'ascendant scientifique du médecin, la «neutralité affective» (Parsons) qu'il s'impose et les exigences 
professionnelles des soignants ou des enseignants - dès lors que les incertitudes sont terribles, que la lassitude est là et la culpabilité jamais loin - semblent insupportables : «La substitution de l'autorité anonyme à l'autorité ouverte s'exprime dans toutes les sphères de la vie » (Fromm 53). La frontière, toujours incertaine entre autorité et autoritarisme, provoque rapidement fermeture, refus, isolement, etc. et stratégies de contournement.

Ces conflits fréquents avec les normes et pratiques professionnelles, sur les questions éthiques, de justice et d'équité, prennent une autre forme avec les institutions et les administrations, compliquant «les rapports entre solidarité publique et privée » (Pennec 248), les partages de responsabilité et d'autorité, les répartitions de charges entre famille et protection sociale. Ce que rapportent de nombreux parents des propos échangés avec le corps médical, renvoie à l'interactionnisme (Strauss) et à la nécessité fréquente de négocier. Pardelà le colloque singulier médecin-malade, les relations en situation d'incertitude sont complexes et les protagonistes du sanitaire, du social et du médico-social sont nombreux.

L'écoute attentive des mots employés, les angoisses sous-jacentes à certains propos et la violence contenue renvoient à Michel Foucault et à Gilles Deleuze quant à l'enfermement et au contrôle de ceux qui entravent l'ordre social bourgeois. Par leur refus de la mise en établissement pour faire respecter leurs enfants, les parents ont conscience d'empêcher qu'on efface le malaise de la lenteur, voire du désordre. Ils remettent en cause les rythmes trépidants, valorisent l'aide adaptée, le repos et certaines activités ordinaires. Ils luttent contre la « subtile élimination » (Prayez) et contestent l'« idéologie du dépassement de la fragilité » (Marzano) selon laquelle la lenteur, la non-performance sont intolérables.

Faire une place à des personnes différentes, les encourager à affronter le regard, c'est reconnaître tout le poids de ce dernier, en mesurer et souligner la force et la dangerosité : « il ne sert pas seulement à la vision mais aussi à la communication. [...] il est la clé de l'échange, c'est par lui que s'engagent les relations. [...] Le regard est le précurseur du langage [...] il y a de ces regards intrusifs ou méprisants, qui interdisent ou qui font mal; il y a aussi des absences de regard, qui traversent les êtres, donnant un sentiment d'inexistence » (Marcelli).

Les tensions entre aspirations nouvelles et inégalités des chances renvoient aux distorsions relevées par Robert K. Merton entre structure culturelle et structure sociale et aux mouvements «divergents» conduisant aux déviances évoquées par Howard S. Becker : parents et enfants recherchent l'autonomie pour les personnes handicapées mais la société, en vue de les protéger, impose ses normes, qui limitent les moyens ou ne permet pas de les adapter. Ce paradoxe est souligné par Charles Gardou (Fragments) : « d'un côté, une fièvre de modernité et d'avenir, comme dans le secteur des sciences, des techniques et de la 
communication ; de l'autre, une résistance, voire une immobilité dans les archaïsmes, s'agissant du regard porté sur nos pairs touchés par un handicap ». L'affirmation respectueuse mais ferme des choix parentaux se fonde sur la légitimité qui les autorise à un regard critique sur les normes en vigueur. Leur militantisme mesuré mais déterminé renvoie à l'observation de Philippe Ariès en 1979 : «L’idéologie soigne les handicapés, le comportement spontané les exclut ». Même après la loi de 2005 et l'évolution des mentalités, entre les principes et le quotidien, les écarts restent nombreux et importants.

Pour amener leurs enfants à une forme d'autonomie sécurisée, les parents retrouvent leur «capacité à transgresser»: ils contestent, argumentent, exploitent les liens et les interstices, les contradictions des politiques publiques, les imprécisions administratives. Pour imaginer et mettre en place une autre vie, espérer en restant lucide et refuser l'isolement, ils créent et renforcent les repères, resserrent les liens familiaux, expliquent et nouent des relations amicales, s'appuient et se font aider par les professionnels et les associations.

Cette sociabilité beaucoup travaillée construit et étaye sans cesse leur réflexion et leur volonté de lutter contre le déterminisme dans tous les aspects de la vie sociale. Former à la liberté passe par le refus de l'emprise, de la «domination de groupes» de médecins, enseignants, éducateurs, travailleurs sociaux, la lutte contre «une déconsidération ambigüe de la personne » (Nuss 110) et contre la technicisation médicale. Déterminés par leur objectif, avec et pour leurs enfants, les parents privilégient l'humanité, la proximité et la compréhension plutôt que l'efficacité et la rapidité. Ils déplorent que « les gestionnaires et leaders, aussi bien dans le secteur public que dans le secteur privé associatif ou lucratif, paraissent entraînés dans une même spirale managériale conjuguant économie de moyens, maîtrise des ressources et des dépenses, critère de qualité et évaluation » (Chauvière 189). Ils refusent la promiscuité et les excès d'affectivité, luttent contre les évaluations mal fondées, les méthodes éducatives mal pensées, polluées par le besoin de pouvoir, l'enfermement dans le statut ou la pitié. Plusieurs remarques, en entretiens, rappellent l'analyse de J. Ladsous (60) : «vouloir qu'un enfant qui met l'institution en échec ait un bilan psychométrique peut soit signifier que l'on souhaite évaluer ses compétences pour mieux l'aider, soit qu'on cherche à obtenir une caution 'scientifique' à une décision qui 'arrangerait' tout le monde ».

\section{Apprentissages et savoirs pour l'action}

Les parents se soutiennent, échangent les informations, partagent les solutions efficaces, se préparent et s'entraînent à ne pas se décourager devant les lenteurs administratives, les rejets bureaucratiques, les blocages de budgets. En recherche permanente 
réelle et figurée d' '« une authentique démarche de mise en accessibilité des cadres bâtis et de compensation des handicaps » (Nuss 103), ils sont conscients que «l'innovation suppose le collectif, l'innovation est affaire de réseaux» (Gardien 15). Force de propositions, l'association, présente aux réunions de chantier pour la réhabilitation du complexe, a demandé des ajustements et négocié les hauteurs d'équipements (accessibilité, dangerosité, etc.) et incité les jeunes à choisir leurs couleurs de logement.

Comme leurs illusions ont été mises à mal depuis l'annonce du handicap et à chaque découverte d'autres limites, les parents redoublent de vigilance pour qu'elles ne soient pas aggravées par le contexte, les attitudes et/ou l'ambiance. Ils dévoilent, toujours avec pudeur, la réalité médicale qui s'est imposée il y a quelques décennies et qui se maintient, du fait des fragilités. Ils agissent là où ils en ont le pouvoir, en communiquant, suscitant des confrontations et veillant à créer des liens de proximité. Loin de gommer les spécificités des situations de handicap, ils les expliquent pour que, mieux connues, les frontières s'estompent. $\mathrm{Au}$ fil du temps, ils ont appris à se méfier des solutions toute faites, des naïvetés périlleuses, des situations sans efforts. Identifiés, les obstacles et barrages peuvent devenir contournables et les ressources et fonctionnalités de la technologie, utilisables pour compenser au mieux les manques : «L'alternative réside dans une révolution de la manière de penser et de prendre en compte le handicap » (Gardou, Fragments, 11).

Les parents se conduisent en « réalistes pragmatiques » (Fromm 45) et construisent au quotidien l'avenir. Refusant la «trivialité » au sens littéral du terme, ils n'encombrent pas les autres avec les difficultés de leur vécu pour ne pas ennuyer ou lasser. Ils s'entraînent à « penser contre la bien-pensance » (Gardou, Fragments, 13) mais évitent tout ce qui serait contre-productif. Ils rejettent les doctrines prometteuses autant que les assistances pernicieuses et se défient des postures antalgiques, qui deviendraient vite impostures. La perspective de « l'après-nous » est toujours sous-jacente.

Pour faire accepter le handicap comme faisant partie de la personne, requérant bienveillance et fermeté, le travail d'interprétation est quotidien. Par-delà la médiation, la traduction qui transforme l'étranger en familier, il s'agit d'éduquer, d'expliquer, de convaincre pour aider tout un chacun à comprendre et trouver l'intérêt du « vivre avec » les situations handicapantes et les personnes handicapées. Le dispositif rappelle « la vigie », telle que l'évoquent des chercheurs canadiens, articulée sur le partenariat association-chercheurs «dans l'objectif de produire des connaissances et de les mettre en pratique » (Boucher et Fiset 105). 
Si avec Michel Wieviorka, l'on admet que «l'altérité est aussi une question qui renvoie à l'existence de collectivités, de groupes plus ou moins instables, et dont les membres peuvent souhaiter, éventuellement, que leur identité soit reconnue dans l'espace public »(9), on peut considérer que les préoccupations et activités des parents sont en permanence orientées vers l'aide qu'ils reconnaissent utile, efficace pour que leurs enfants se fassent ou trouvent une "place », s'intègrent dans la cité, accèdent aux droits et à une citoyenneté. «Prendre en compte la personne dans son intégralité et son intégrité » (Nuss 110) est complexe, cela demande beaucoup de réflexion et des efforts de recul répétés. Le sort commun de la trisomie 21 ne gomme en rien les particularités de personnalités, les spécificités de parcours familiaux, les habitudes culturelles. Or, l'action commune passe, le plus souvent, par des démarches individuelles, liées au cas de chacun, ce qui induit en permanence « un arrangement mutuel respecté » (Fromm 48).

Imposer ce «vivre avec» exige une lutte de tous les instants pour la «désinsularisation» (Gardou, Fragments) et une meilleure perception de ce que les personnes en difficultés nous enseignent sur nous-mêmes et nous apportent : d'autres représentations du monde, d'autres rythmes, d'autres interrogations. Inciter aux questions et à la réflexion sur les conditions et modalités de cet apport exige des savoir-faire que les parents sont conscients de perfectionner chaque jour : «ce serait à refaire, je n'expliquerais plus les choses comme cela », « ce n'était pas comme cela qu'il fallait présenter le dossier »... Le poids du relationnel - des mots, des gestes, des regards... - s'est imposé à eux, ils en connaissent négativement et positivement le « rôle d'amplificateur » (Gardou).

Aux plans psychologique et sociologique, leurs savoir-faire d'expériences, les apprentissages informels sur longues périodes leur ont montré qu'un développement « autrement» de la personne donne le sentiment d'exister, de «bien-vivre», conduit à s'ouvrir aux autres : capacité à être aimable puisqu'on se sent aimé. C'est le chemin vers la construction de relations d'amitié, la prise de distance pour être libre et la résistance aux sollicitations et aux personnes.

Tous ces principes sous-tendent la qualité des relations de voisinage dans un ensemble immobilier comme pour la vie de quartier ou de village. Ils sont aussi au fondement du bien-être au travail, si préoccupant dans certaines organisations, dont les recherches de standardisation et d'efficacité ont nié les bienfaits de la diversité. Les propos de Stiker (Corps) sont à nouveau présents : la vie en compagnie de tout type de handicap, en particulier la déficience intellectuelle, nous rend «déboutés de nos assurances et de nos références mimétiques et de nos visions normées ». Comme les vulnérabilités des personnes 
handicapées ou vieilles, immigrées, sans emploi, sans domicile, renvoient «une image déformée de nous-mêmes jusqu'à détruire le sentiment intime de notre identité... » (AssoulyPiquet 1994), l'apprentissage donc la confrontation sont nécessaires. C'est à cette « transgression constructive» de tous ceux qui acceptent et peuvent comprendre, que les parents appellent.

Leurs efforts variés et dans la durée, pour faire progresser leur enfant, constituent une véritable expérience du handicap, un savoir-faire empirique, acquis chemin faisant, qui les légitime et les conduit à poser divers questionnements qu'ils renvoient aux « autres », aux gens «ordinaires»: qu'en faisons-nous ? Que pouvons-nous en apprendre ? Jusqu'où et comment faire ou donner confiance?

Cette découverte renforce leur refus de l'emprise, leur tendance à la relativisation des normes et des avis. Pour progresser, ils se renseignent, remettent en cause les règles, habitudes et contraintes organisationnelles, locales et nationales ou au moins, en perspective d'autres systèmes, pays, époques. Cela leur suggère des amodiations ou assouplissements et les incite à demander des adaptations de méthodes.

La structuration empirique et progressive du groupe leur a fait franchir les étapes évoquées par Claude Giraud «entre le collectif comme agrégat, le collectif comme conscience sociale, le collectif comme communauté et le collectif comme entrecroisement de transactions » (80). Peu à peu, ils mesurent que ce sont des «réalités différentes », que la transmission des retours d'expériences ne reste porteuse qu'au moins en partie formalisée, que les mises en commun, échanges/confrontation sans conservation de traces perdent beaucoup d'intérêt. De là, les tentatives de définitions de lignes de conduite, l'application de méthodes qui déclenchent, en interne comme chez les partenaires ou les institutionnels, des observations, critiques et de nouveaux tâtonnements /ajustements.

La conscience de la fragilité de leur projet les rend d'autant plus forts car motivés. Lorsqu'ils souhaitent et requièrent amodiation, assouplissement, adaptation des méthodes, ils travaillent plutôt en collaboration - négociation - partenariat qu'en imposition. Les relations, échanges qui leur sont nécessaires touchent tant aux conditions de la vie privée, qu'au sens du travail, de l'habitat, de la ville, des divertissements, de la communication. Ce sont de nombreux aspects du lien social qu'ils revisitent pour lutter contre «la domination consensuelle de la raison instrumentale dans les secteurs les plus divers de la société » (Spurk 43). Comme la plupart des collectifs présents dans le domaine du handicap, ils demandent pour et avec leurs enfants «l'application concrète de leurs droits fondamentaux » et relèvent 
que «l'exercice de la citoyenneté est décidément, pour certains, un rapport de force en démocratie » (Gardien 246).

La recherche de «l'autodétermination» qu'ils évoquent pour leurs enfants, correspond à une oscillation entre rêve et espoir, dont l'inquiétude n'est jamais absente. L'autodétermination est complexe dans son contenu, ses formes, ses objectifs, variable dans le temps, l'espace, tributaire des situations. Fondée sur des principes philosophiques, utilisée en droit international, elle prend un sens à la fois plus étroit et plus flou dans ce contexte. Pardelà les aides financières et matérielles, les parents souhaitent une remise en question des places et la prise en considération de ce que chacun peut apporter à la société. Les «capabilités» (Sen) des personnes, les transmissions parentales de savoirs et les caractéristiques sociologiques des familles influent sur l'aptitude à formuler des choix, à exercer ses droits (argent, sexualité, etc.), à comprendre et respecter ses obligations. L'autodétermination se réfère donc à des éléments et des niveaux divers, selon le type/les modalités, la profondeur, la durée et les contenus de l'accompagnement (parents/famille, professionnel, institutions, etc.), avec (en tous cas) le courage comme «Vertu centrale» (Fleury). Ce que les parents et certains chercheurs (Nuss ; Caouette) évoquent au travers de l'autodétermination, est appelé par d'autres «autonomie sécurisée » ou « raisonnée ». Ce cheminement, cet espoir représentent un réel défi, relevé par une minorité déterminée et agissante, qui par une éducation attentive et adaptée, est parvenue à faire progresser de jeunes handicapés jusqu'à les insérer dans la « vie ordinaire ». Espérant cantonner leur rôle actuel et futur à l' "équilibration » et aux «avertissements », ces parents, dans une distance très construite, continuent à veiller et essaient en permanence de voir plus loin pour oser formuler des projets.

Leurs cumuls de transgressions «chemin faisant» pour lutter contre les catégorisations et l'enfermement, renvoie à la «destruction créatrice », facteur d'innovation de Schumpeter ; on est dans une forme de «désorganisation » sociale (Thrasher), au sens d'un état transitoire caractérisé par la mise en place d'organisations spontanées dans les « interstices» de «la société en mouvement» (Sainsaulieu). Cette démarche courageuse et raisonnée interpelle, placée sous la « double contrainte de l'ambiguïté des pratiques sociales et de l'antagonisme des valeurs » (Van Haecht 5), elle interroge les métiers, le lien et la protection sociale sous des angles moins institutionnels, plus différenciés.

\section{Bibliographie}

Ariès, Philippe. Un historien du dimanche. Paris : Seuil, 1979. 
Assouly-Piquet, Colette et Francette Berthier-Vittoz. " Regards sur le handicap ». Hommes et perspectives/épi. Paris : n.p., 1994.

Beck, Ülrich. La société du risque : sur la voie d'une autre modernité. Paris : Aubier, 2001.

Becker, Saul Howard. Outsiders, Etude de la sociologie de la déviance. Paris : Métaillé, 1985.

Boucher, Norman et Daniel Fiset. «La vigie : une pratique novatrice de défense collective des droits humains ». Des innovations sociales par et pour les personnes en situation de handicap. Dir. E. Gardien. Toulouse : Érès, 2012. 106-117.

Caouette, Martin. «Étude descriptive des pratiques d'intervention en CRDITED favorisant l'autodétermination d'adultes présentant une déficience intellectuelle : la perspective des intervenants ». Thèse de doctorat en psychoéducation UQTR : avril 2014.

Chauvière, Michel. Trop de gestion tue le social. Essai sur une discrète chalandisation., Paris : La Découverte, 2010.

Chiflet, William. Sois Bègue et tais-toi. Paris : Archipel, 2014.

Cresson, Geneviève. Les parents d'enfants hospitalisés à domicile. Leur participation aux soins. Paris : Harmattan, 2001.

Deleuze, Gilles. « Post-scriptum sur les sociétés de contrôle ». L'autre journal 1 (1990).

Fleury, Cynthia. La fin du courage. Paris : Fayard, 2010.

Flis-Trèves, Muriel. «L'autre, le semblable, le différent ». L'Autre, le semblable, le différent. Dir. René Frydman et Muriel Flis-Treves. Paris : PUF, 2014. 1-2.

Foucault, Michel. Surveiller et punir, naissance de la prison. Paris : Gallimard, 1975.

Fromm, Eric. L'art d'être. Paris : Desclée de Brouwer, 2000.

Frydman, René et Muriel Flis-Treves dir. L'autre, le semblable, le différent. Actes du colloque « GYnécologie XIII PSYchanalyse ». Paris : PUF, 2014.

Gardien, Ève, dir. Des innovations sociales par et pour les personnes en situation de handicap. Toulouse : Érès, 2012.

Gardou, Charles. Fragments sur le handicap et la vulnérabilité, Pour une révolution de la pensée et de l'action. Ramonville Saint Agne : Érès, 2009.

---, dir. Professionnels auprès des personnes handicapées. Ramonville Saint Agne : Érès, 2000.

--- et Denis Poizat, Denis, dir. Désinsulariser le handicap. Quelles ruptures pour quelles mutations culturelles? Ramonville Saint Agne : Érès, 2007. 
Giraud, Claude. " A propos de l'indifférence et de l'envie ». Les «creux» du social. De l'indéterminé dans un monde se globalisant. Dir. B. Ganne et Glysi-Safa. Paris : Harmattan, 2005. 79-98.

Goffman Erving. Stigmate. Les usages sociaux des handicaps. Tr. A. Kihm, Paris : Minuit, 1975.

Gossot, Bernard et Claude Mollo. Scolariser les jeunes handicapés. Les Rapports de l'Inspection Générale de l'Education nationale (IGEN). Paris : CNDP / Hachette / Documentation française, 1999.

Hastings, Michel, Loïc Nicolas et Cédric Passard, Cédric, dir. Paradoxes de la transgression. Paris : CNRS, 2012.

Ionescu, Serban. «Les mécanismes de défense ». Le moi, du normal au pathologique. Dir. Gaëtane Chapelle. Auxerre : Sciences humaines, 2004. 45-53.

Jodelet, Denise, dir. Les représentations sociales. Paris : PUF, 2003.

Kaufmann, Jean-Claude. Pour une sociologie de l'individu. Paris : Nathan, 2001.

Korff-Sausse, Simone. Figures $d u$ handicap. Mythes, arts, littérature, Paris : Petite bibliothèque Payot, 2010.

---, dir. La vie psychique des personnes handicapées. Ce qu'elles ont à dire, ce que nous avons à entendre. Coll. « Connaissances de la diversité ». Toulouse : Érès, 2009.

Laloux, Eléonore, avec Yann Barte. Triso et alors ! Paris : Max Milo, 2014.

Lecomte, Jacques. «Le 'coping', ou comment nous faisons face au stress ». Le moi, du normal au pathologique. Dir. Gaëtane Chapelle. Auxerre : Sciences humaines, 2004. 53-54.

Lipovetsky, Gilles. Le bonheur paradoxal. Coll. « Folio essais ». Paris : Gallimard, 2006.

Marcelli, Daniel. Les yeux dans les yeux, l'énigme du regard. Paris : Albin Michel, 2005.

Marzano, Maria Michela. Contrat de défiance. Paris : Grasset, 2010.

Merton, Robert King. "Social Conformity, Deviation and Opportunity-structures ». American Sociological Review 24 (1959).

Nuss, Marcel, avec V. Cohier-Rahban. L’identité de la personne "handicapée». Paris : Dunod, 2011.

Parsons, Talcott Elger. The Social System. New York : The Free P, 1951.

Pelluchon, Corine. L'autonomie brisée. Bioéthique et philosophie. coll. Quadrige, Paris : PUF, 2014.

Pennec, Simone. «La souffrance des proches : ajustements négociés entre ses propres valeurs et normes de santé, celles de la famille et celles des professionnels ». Normes et 
valeurs dans le champ de la santé. Dir. F. X. Schweyer, S. Pennec, G. Cresson et F. Bouchayer . Paris : ENSP, 2004. 237-250.

Prayez, Pascal et Corinne Sliwka, dir. Distance professionnelle et qualité du soin. Fonction Cadre de santé. Paris : Lamarre, 2009.

Sainsaulieu, Renaud. Des sociétés en mouvement. La ressource des institutions intermédiaires. Paris : Desclée de Brouwer, 2001.

Sausse, Simone. Le miroir brisé. L'enfant handicapé, sa famille et le psychanalyste. Paris : Calmann Levy, 1996.

Scelles, Régine. "Intégration scolaire : ce que les enfants disent ». La vie psychique des personnes handicapées. Ce qu'elles ont à dire, ce que nous avons à entendre. Dir. Simone Korff-Sausse. Toulouse : Érès, 2009. 115-130.

Schumpeter, Joseph. Capitalisme, socialisme et démocratie. Paris : Payot, 1951.

Sen, Amartya. Éthique et économie. Paris : PUF, 2008.

Spurk, Jan. Malaise dans la société. Soumission et résistance. Lyon : Parangon, 2010.

Stiker, Henri-Jacques. Corps infirmes et sociétés. Paris : Aubier, 1982.

---, José Puig et Olivier Huet. Handicap et accompagnement. Nouvelles attentes, nouvelles pratiques. Paris : Dunod, 2009.

Strauss, Anselm. La trame de la négociation. Sociologie qualitative et interactionniste. Dir. I. Baszanger. Paris : Harmattan, 1992.

Styron, William. Face aux ténèbres. Paris : Gallimard, 1993.

Thrasher, Frederic. The Gang : A Study of 1,313 Gangs in Chicago. Chicago : U of Chicago P, 1927.

Valadier, Paul. Eloge de la conscience. Coll. « Esprit», Paris : Seuil, 1994.

Weiss. Isabel. L'interprétation. Paris : Ellipses, 2002.

Wievorka, Michel. «L'autre le semblable, le différent ». L'autre, le semblable, le différent. Dir. René Frydman et Muriel Flis-Treves. Colloque «GYnécologie XIII PSYchanalyse ». Paris : PUF, 2014. 3-13.

\footnotetext{
NOTE

${ }^{1}$ M. Caouette, Ph. D., ps.ed., professeur, Département de psychoéducation, Université du Québec à TroisRivières, R. Plichon doctorant en aménagement du territoire, U.A., LEM Artois UMR 8179 CNRS, A-C. Taillandier, MCf, U.Artois, Arras LEM Artois UMR CNRS 8179 CNRS, Z. Zéroulou, MCf Lille 1, CLERSE UMR CNRS 8019.
} 